\title{
Transferring brain-computer interfaces beyond the laboratory: Successful application control for motor-disabled users
}

\author{
Robert Leeb ${ }^{\mathrm{a}, *}$, Serafeim Perdikis ${ }^{\mathrm{a}}$, Luca Tonin ${ }^{\mathrm{a}}$, Andrea Biasiucci ${ }^{\mathrm{a}}$, Michele \\ Tavella $^{\mathrm{a}}$, Marco Creatura ${ }^{\mathrm{a}}$, Alberto Molina ${ }^{\mathrm{a}}$, Abdul Al-Khodairy ${ }^{\mathrm{b}}$, Tom \\ Carlson $^{\mathrm{a}}$, José d. R. Millán ${ }^{\mathrm{a}}$ \\ ${ }^{a}$ Chair in Non-Invasive Brain-Machine Interface, Center for Neuroprosthetics, École \\ Polytechnique Fédérale de Lausanne, Station 11, CH-1015 Lausanne, Switzerland \\ ${ }^{b}$ Clinique Romande de Réadaptation-Suvacare, Avenue du Grand-Champsec 90, CH-1950 \\ Sion, Switzerland
}

\begin{abstract}
Objectives: Brain-computer interfaces (BCIs) are no longer only used by healthy participants under controlled conditions in laboratory environments, but also by patients and end-users, controlling applications in their homes or clinics, without the BCI experts around. But are the technology and the field mature enough for this? Especially the successful operation of applications -like text entry systems or assistive mobility devices such as tele-presence robots- requires a good level of BCI control. How much training is needed to achieve such a level? Is it possible to train naïve end-users in 10 days to successfully control such applications?

Materials and methods: In this work, we report our experiences of training 24 motor-disabled participants at rehabilitation clinics or at the end-users' homes, without BCI experts present. We also share the lessons that we have learned through transferring BCI technologies from the lab to the user's home or clinics.

Results: The most important outcome is that fifty percent of the participants achieved good BCI performance and could successfully control the applications (tele-presence robot and text-entry system). In the case of the

*Corresponding author. Tel.: +41 21693 6969; fax: +41 21693 5307; web: http://cnbi.epfl.ch/.

Email address: robert.leeb@epfl.ch (Robert Leeb)
\end{abstract}


tele-presence robot the participants achieved an average performance ratio of 0.87 (max. 0.97) and for the text entry application a mean of 0.93 (max. 1.0). The lessons learned and the gathered user feedback range from pure BCI problems (technical and handling), to common communication issues among the different people involved, and issues encountered while controlling the applications.

Conclusion: The points raised in this paper are very widely applicable and we anticipate that they might be faced similarly by other groups, if they move on to bringing the BCI technology to the end-user, to home environments and towards application prototype control.

Keywords: Brain-computer interface (BCI), electroencephalogram (EEG), motor imagery, application control, end-user, technology transfer

\section{Introduction}

The idea and the technology to control machines, not by manual operation but by mere "thinking" is called the Brain-computer interface (BCI) [1]. Most often the electrical activity is recorded from the brain non-invasively by means of the electroencephalogram (EEG). Control features are extracted from this activity, which can be used by disabled people to establish a new communication channel between the human brain and a machine. Several BCI prototypes have been demonstrated over the last decade [2] for applications such as (i) communication and control, e.g. writing on a virtual keyboard $[3,4]$ or browsing the Internet $[5,6]$, (ii) the control of wheelchairs $[7,8]$ or robots [9, 10], and (iii) computer games for healthy users [11, 12] or virtual reality applications $[13,14]$.

Most of the applications presented in the literature tend to be either software oriented, like mentally writing text via a virtual keyboard on a screen, or more hardware oriented, like controlling a small mobile robot. Typically such applications require a relatively good and precise control channel to achieve performances comparable to healthy people using conventional interfaces. However, current day BCIs offer low information throughput and are insufficient for the full dexterous and sustained control of these complex applications. Therefore, techniques like shared control or context awareness can enhance the interaction to reach a similar level, compensating for the fact that BCI is not a perfect control channel [15]. In such a control scheme, the responsibilities and efforts are then shared between the user in giving 
high-level commands and the system in executing fast and precise low-level interactions. Furthermore, modern human-computer interaction (HCI) principles can explicitly take into account the noisy and delayed nature of the BCI control signals to adjust the dynamics of the interaction as a function of the reliability of user's control capabilities [4].

Although most of the prototypes and applications target disabled users, the vast majority of the published work is based on the analysis of data from healthy participants. Nevertheless, there have been some success stories with patients and end-users $[3,16]$, although most of these works intensively require the BCI experts to host the participants at the research labs or go to end-users homes. Therefore, it is crucial for the field to cross another frontier, by letting caregivers or therapists support the end-users in the use of BCIs without (or with minimum) supervision or interference from BCI experts. Our plan was for caregivers to undertake the whole job of BCI setup and operation, while the BCI experts provide (if needed at all) troubleshooting advice via telephone or via remote support platforms (like "tele-monitoring" [17]).

In this paper, we report our experience, and the problems we encountered, while transferring our BCI from the lab to clinics and to end-users' homes, and while moving from simple BCI control towards successfully control of applications. We started with naïve, severely motor-disabled users, teaching them first to achieve BCI control, evaluating the performance through online BCI experiments and finally controlling two applications (either a writing application for communication or a robotic tele-presence platform for assistive mobility). The aim was to do this in 10 days (spread over a number of weeks), working together with a therapist at a rehabilitation clinic and without any BCI experts on site. All the points raised and discussed in this paper are widely applicable and we anticipate that they might be faced similarly by other groups, moving on to bring the BCI technology to the end-user, to home environments and towards application prototype control.

\section{Materials and methods}

In this Section we first describe the participants, the training process and the experimental paradigm to achieve BCI control, then the signal processing and machine learning methods to identify suitable brain features, through which the participants delivered the BCI commands during the recordings. Furthermore, we present: the hardware infrastructure needed to perform 
this training at the end-user's location; the two tested applications; and the applied evaluation criteria.

\subsection{Participants and training locations}

Twenty-four end-users aged $42.7 \pm 14.1$ years ( 3 female) have been trained at the various out-of-the-lab locations (either at clinics, assistive technology support centers or users' homes in Switzerland, Germany and Italy), without BCI experts present (Fig. 1.A). They have participated once or twice a week (sometimes only every other week) for up to 3 hours per day, with a maximum number of 10 sessions (recording days). The end-users are affected by different levels of myopathy, spinal cord injury, tetraglegia, amputation, spino-cerebellar ataxia or multiple sclerosis, but none of the participants have mental deficits. Details for each end-user are given in Table 1.
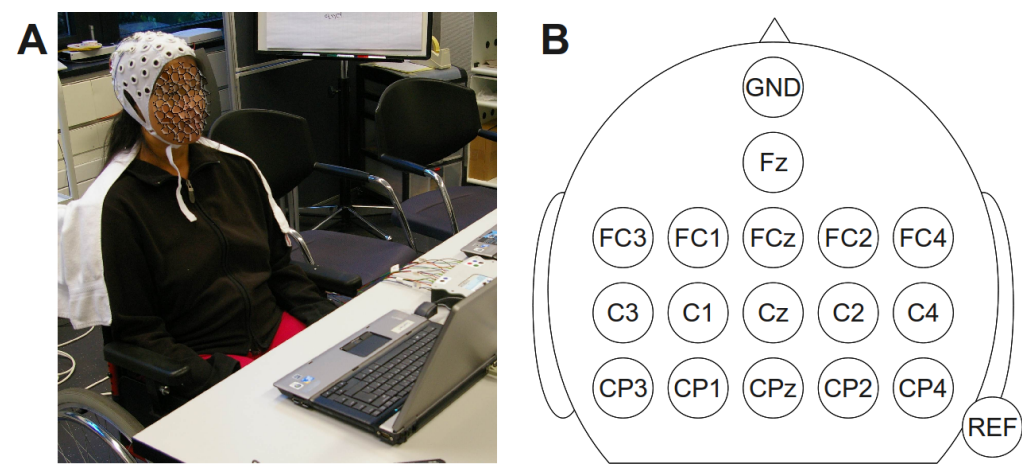

Figure 1: (A) End-user at a clinic while operating the BCI. (B) EEG electrode locations used over the motor cortex.

\subsection{Training process}

In the presented study, a BCI based on motor imagery (MI) is used. MI is described as the mental rehearsal of a motor act without any overt motor output [18], which involves similar brain regions to those which are used in programming and preparing such real movements [19, 20]. The imagination of different types of movements (e.g. right hand, left hand or feet), results in an amplitude suppression (known as event-related desynchronization, ERD [21]) or in an amplitude enhancement (event-related synchronization, ERS)) of Rolandic mu rhythm $(7-13 \mathrm{~Hz})$ and the central beta rhythm (13-30 Hz) recorded over the sensorimotor cortex of the participant [22]. 
Table 1: Details of end-users who participated in the experiment, including the time since the injury or diagnosis and the age both in years. Participants which years are marked by "-" are congenital-hereditary. Note: To increase the readability of the paper, the participants have been ordered in descending order to their final BCI online performance (see Section 3.2) independently of the date of recording.

\begin{tabular}{lllll}
\hline ID & Sex & Medical condition & Time & Age \\
\hline P1 & M & Tetraplegia C5-C6 & 23.0 & 44.4 \\
P2 & M & Muscular dystrophy (Duchenne) & - & 18.4 \\
P3 & M & Tetraplegia C3 & - & 42.4 \\
P4 & F & Myopathy & 4.2 & 35.4 \\
P5 & M & Spinal cord injury C7 & 10.3 & 59.8 \\
P6 & M & Tetraplegia C6 & 22.5 & 47.8 \\
P7 & M & Tetraplegia C6 & 24.4 & 42.1 \\
P8 & M & Tetraplegia C6 & - & 30.8 \\
P9 & M & Myopathy: spinal amyotrophy-type 2 & 9.3 & 32.0 \\
P10 & M & Tetraplegia C4 & 4.2 & 51.5 \\
P11 & M & Incomplete locked-in syndrom & 5.7 & 29.2 \\
P12 & M & Tetraplegia C5 & 3.3 & 38.3 \\
P13 & M & Amyotrophic lateral sclerosis & - & 27.7 \\
P14 & M & Cerebral palsy & 4.2 & 58.2 \\
P15 & M & Amyotrophic lateral sclerosis & 1.2 & 70.2 \\
P16 & F & Left shoulder-hand syndrome (complex regional & & \\
& & atrophy) following fracture of the left wrist, can- & & \\
P17 & F & not use fully her upper arm & - & 62.1 \\
P18 & M & Tetraplegia C5 & 3.2 & 52.8 \\
P19 & M & Amputation at upper third of the left fore-arm, & 5.5 & 29.2 \\
& & amputation of left lower limb at knee level (phan- & & \\
P20 & M & tom limbs) & & 51.5 \\
P21 & M & Spino-cerebellar ataxia & - & 30.5 \\
P22 & M & Tetraplegia C5 & 14.4 & 32.6 \\
P23 & M & Tetraplegia C5, after Guillain-Barré disease & 27.9 & 51.4 \\
P24 & M & Tetraplegia & 7.2 & 63.4 \\
\hline & & & &
\end{tabular}
the sensorimotor cortex: Fz, FC3, FC1, FCz, FC2, FC4, C3, C1, Cz, C2, 
C4, CP3, CP1, CPz, CP2 and CP4 according to the international 10-20 system with reference on the right ear and ground on $\mathrm{AFz}$ (see Fig. 1.B). The EEG is recorded using a 16-channel g.USBamp (g.tec medical engineering, Schiedelberg, Austria) system at $512 \mathrm{~Hz}$, band-pass filtered between $0.1 \mathrm{~Hz}$ and $100 \mathrm{~Hz}$ and a notch filter is set at the power line frequency of $50 \mathrm{~Hz}$.

Before being able to use a BCI, participants have to go through a number of steps to learn to voluntarily modulate the EEG oscillatory rhythms by performing MI tasks. Furthermore, the BCI system has to learn what the participant-specific patterns are. In our case, all participants start by imagining left hand, right hand and feet movements during a number of calibration recordings. Afterwards, the EEG data is analyzed (see Section Appendix A), a classifier is then built for each pair of MI tasks that the user has rehearsed and the pair of tasks which shows highest separability and is most stable, is used for BCI control for that particular user (online experiments). If participants achieve good online control (see Section 2.4 for the performance criteria), they are allowed to test the application prototypes (applications, see Section 2.3). The time-line of the different stages is illustrated in Fig. 2. More details about the experimental paradigm, signal processing and machine learning (feature extraction, feature selection, classification and evidence accumulation) and the feedback are given in Appendix A.

One aim of this study is to complete the whole training and testing process within 10 sessions (maximum allowed time) at a rehabilitation clinic or users home, otherwise the training process is stopped and the participant is dropped from the study. All experiments are conducted according to the declaration of Helsinki and the study is approved by the local ethics committee. All participants are asked to give written informed consent before participating in the study. Furthermore, they are explicitly instructed that they can exit the study at any time without giving any reason.

\subsection{Application prototypes}

Two BCI applications are chosen to be tested here: first an assistive mobility application represented by a tele-presence robot and second a communication application represented by a text-entry system. Real applications are always more demanding for the participants, since besides the increased workload and the split attention between the BCI feedback and the application control (dual task), it is also necessary to perform the requested BCI action with certain temporal precision, especially in case of the robot. Therefore, the user will be supported in accomplishing the task by human com- 


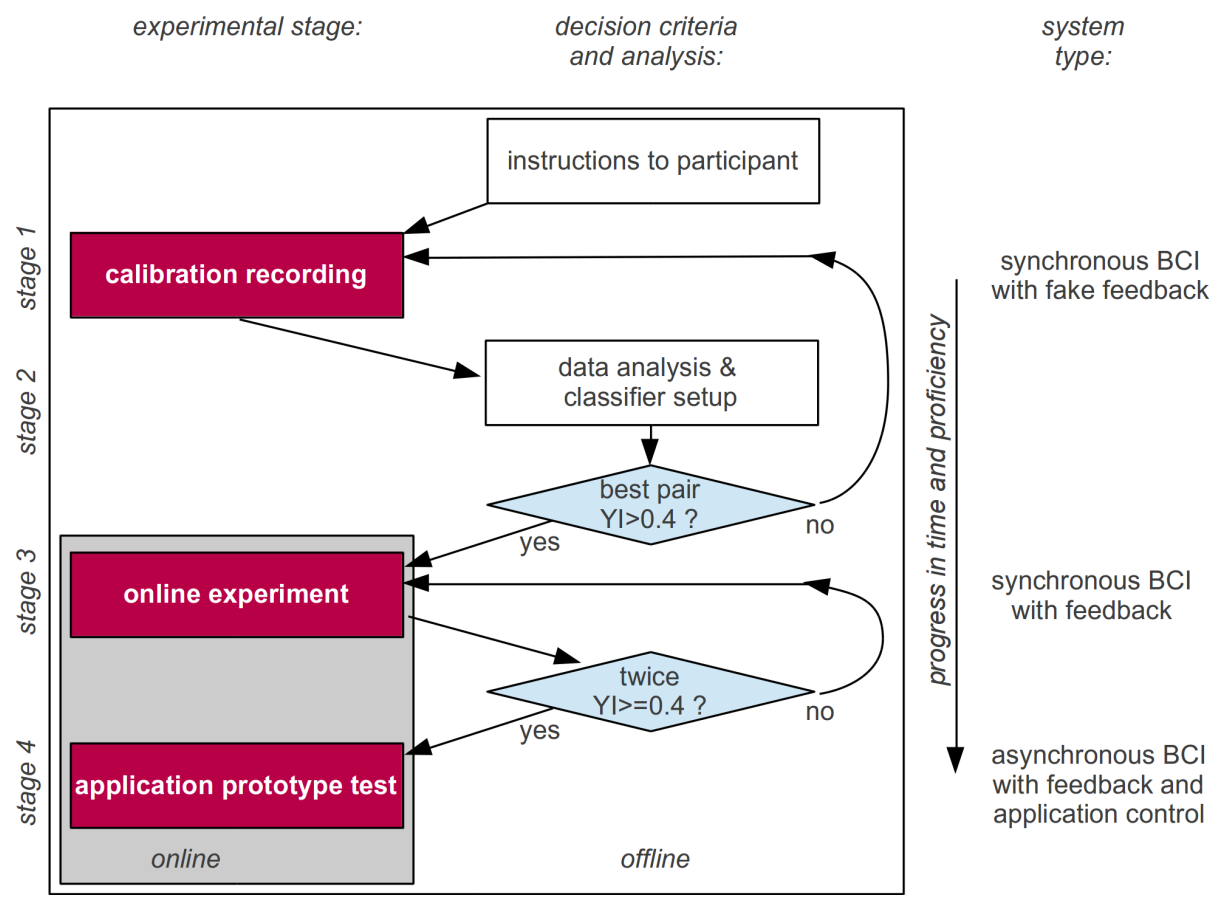

Figure 2: Different stages of the BCI training. Each participant starts with a calibration (offline) recording. Data analysis, identification of the best motor imagery (MI) pair and classifier setup are performed, before online BCI experiments can be conducted. If a good and stable BCI performance (measured as Youden index, YI) can be achieved, the participants are allowed to test the applications. Depending on the performance of the participant, some steps can be repeated several times.

puter interaction, context awareness and shared control techniques, which are specified in more detail below.

\subsubsection{Application: Assistive mobility}

In this work the Robotino ${ }^{\mathrm{TM}}$ robot by FESTO (Esslingen, Germany) was used, which is a small circular mobile platform (diameter $36 \mathrm{~cm}$, height $65 \mathrm{~cm})$. The robot is equipped with nine infrared sensors that can detect obstacles at up to $\sim 30 \mathrm{~cm}$ distance and a webcam that can also be used for obstacle detection. Furthermore, a notebook with a camera is added on top of the robot for tele-presence purposes (see Fig. 3.A), so that the participant can interact with the remote environment via Skype ${ }^{\mathrm{TM}}$, which was not part of the formal evaluation, except seeing the video stream from the robot for 
navigation purposes.

Using the 2-class BCI, the participant remotely controls the robot turning to the left or to the right to reach several targets within an office environment (four predefined target positions). The space contains natural obstacles (i.e. desks, chairs, furniture, people) in the middle of the pathways (see Fig. 3.B). Importantly, participants have never been in such an environment. In addition, the participant can intentionally decide not to deliver any mental commands to maintain the default behavior of the robot, which consists of moving forward and avoiding obstacles with the help of a shared control system using its on-board sensors. The participant sees the video-transmission from the tele-presence camera of the robot in parallel to the BCI feedback.

The same paths are driven twice, once controlled with the BCI in combination with shared control and once as a baseline recording, directly controlled via manual button presses without shared control (i.e. any remaining muscular activities of the participants, like hand or head movements). These two conditions are the necessary subset identified in [23] with non-disabled participants to compare BCI with manual control. The shared control implementation is based on the dynamical system concept coming from the fields of robotics and control theory [24]. Two dynamical systems are created, which control two independent motion parameters: the angular and translational velocities of the robot. The systems can be perturbed by adding attractors or repellors in order to generate the desired behaviors. The dynamical system implements the following navigation modality. The default device behavior is to move forward at a constant speed. If repellors (obstacles) are added to the system, the motion of the device changes in order to avoid the obstacles. The BCI command is handled by adding an attractor to the system, so that the robot starts turning. Other attractors (targets) could be added to support e.g. a docking behavior, but such methods were not used in this study. The current implementation does not support the active stopping or starting of the robot. More information about the robot and the experiment is given in $[23,15]$.

\subsubsection{Application: Text entry}

The second application is a text entry system called BrainTree [25]. It employs the same asynchronous 2-class motor imagery BCI as the main control modality, enabling the user to deliver two types of commands (left/right) by performing two different MI tasks for controlling a binary text-entry. The main novelties of BrainTree lie in the tight integration of inference mecha- 

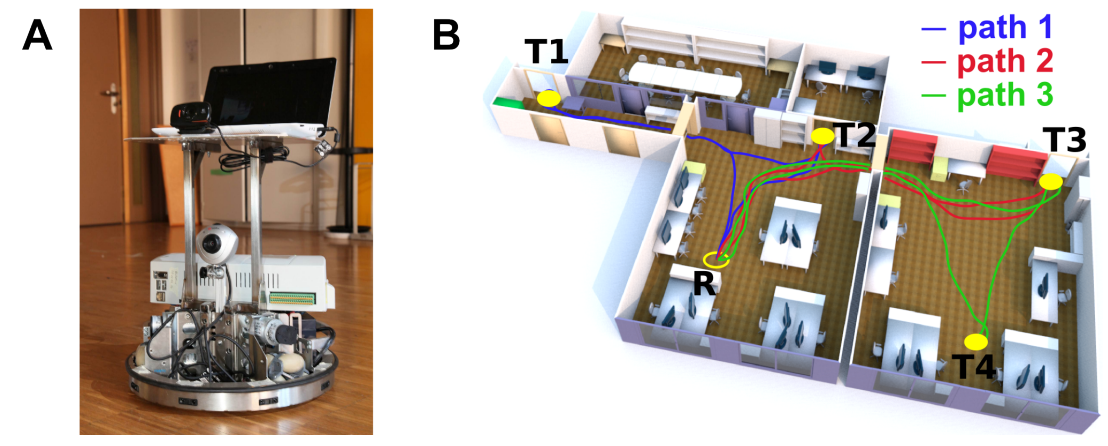

Figure 3: (A) Tele-presence robot. (B) Layout of the experimental environment with the four target positions (T1, T2, T3, T4), start position (R). Lines (path 1, path 2, path 3) indicate possible paths.

nisms with the HCI and the multi-modal control paradigm. Concerning the former, the user observes a simple graphical user interface (GUI, Fig. 4) where all available characters (Latin alphabet including space and backspace) are alphabetically arranged from left to right. This visualization is an intuitive representation, using underlying inference mechanisms based on a Hu-Tucker binary tree [26], which ensures an optimal but not equal number of commands to reach each character (leaf nodes of the tree), based on a learned language model (LM), while preserving the alphabetic order of characters to simplify the visualization.

Regarding the control paradigm, the user's intentions are continuously illustrated in a conventional BCI feedback, where a left/right command is enabled when the feedback bar reaches the threshold. BCI commands result in the associated movement of the red cursor (denoting the current node in the tree), which allows the user to descend the binary tree structure through the BCI, until a leaf node is reached and the associated character is typed. Wrongly written characters can be deleted by selecting the backspace command, which is visible on the far right side of the alphabet in Fig. 4. The orange bubble surrounds the currently available characters (current left/right sub-trees). It further could inform the user about previous erroneous command(s), that need to be "undone" by ascending the tree an appropriate number of times. ${ }^{1}$ The implemented paradigm completely eliminates waiting

\footnotetext{
${ }^{1}$ Based on our user-centered design this fast error correction technique was included and
} 
intervals, thus rendering intentional-non control (INC) skills less important, than in the assistive mobility application. By INC we mean the periods in which the participant is not wanting to deliver any command, e.g. waiting for the next selection step or waiting while a robot is moving forward (e.g. moving down a corridor).

The task of the participant is to "copy-spell" the following four words: hello, internet, email, computer. More information about BrainTree and the experiment is given in [25].

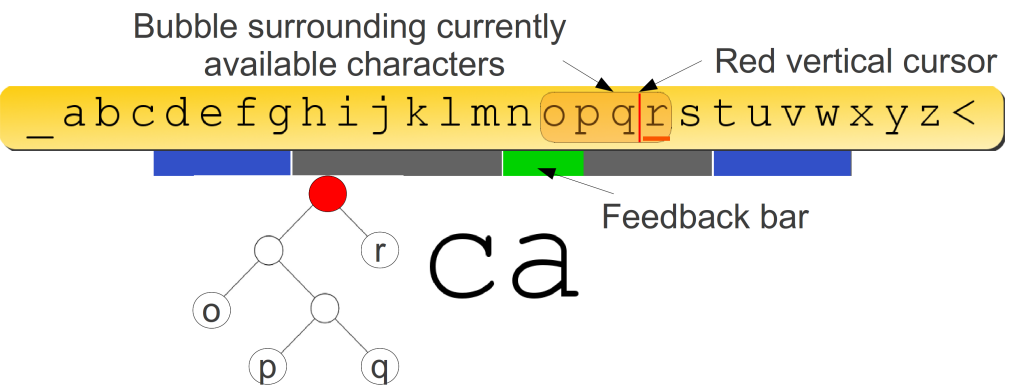

Figure 4: BrainTree Graphical User Interface and associated Hu-Tucker sub-tree while writing the word "car". Prefix "ca" is already typed and the user is navigating towards the character "r". Currently he can select between "opq" with a left command and "r" with a right command, see the orange bubble and position of the red cursor within the alphabet. The BCI feedback bar is shown in green below the alphabet.

\subsection{Evaluation criteria}

BCI performance. The BCI performance of the BCI runs is evaluated using the Youden index (YI, [27]), which is one way to attempt to summarize the true positives rates (TPR) and false positive rates (FPR) in one single numeric value to give an overall diagnostic measure of effectiveness.

$$
\begin{aligned}
Y I & =\text { sensitivity }+ \text { specificity }-1 \\
& =\mathrm{TPR}-\mathrm{FPR} \\
& =\frac{T P}{(T P+F N)}-\frac{F P}{T N+F P}
\end{aligned}
$$

used in later experiments [25]. 
whereby $\mathrm{YI}=1$ means perfect control and 0 equals chance level and TP stands for true positives, $\mathrm{FN}$ for false negatives, FP for false positives and TN for true negatives decisions. In the case of a 2-class synchronous BCI, a YI=1 corresponds to an accuracy of $100 \%$ and a $\mathrm{YI}=0$ to a random accuracy of $50 \%$.

Application assistive mobility. The performances of the robot are reported as the ratio between the distance traveled to reach the targets with BCI control vs. manual control [15], resulting in 1 for the same control performance as with manual buttons, and in 0 or very small values for worse than manual control.

$$
\text { Performance }=\frac{\text { distance }_{\text {Manual }}}{\text { distance }_{\mathrm{BCI}}}
$$

Application text entry. The performances of the BrainTree are reported as the percentage of correctly written characters compared to the total number of written characters (which can consist of correct, wrong and backspace characters) [25], resulting in 1 for perfect and 0 for no control.

$$
\text { Performance }=\frac{\text { characters }_{\text {correct }}}{\text { characters }_{\text {total }}}
$$

\subsection{Remote support infrastructure}

To be able to train the participants alone with their caregivers or therapists we installed a remote support infrastructure. Following the requirements in [17], we used state-of-the-art technologies to setup such a platform, which allowed either to transfer files, to provide communication or to enable a remote takeover in case of technical problems.

A synchronized data folder allowed an automatic transfer (via Unison) of the recorded files from the end-user to the BCI experts via a secured server, and of classifiers or configuration files back to them. Communication via Skype ${ }^{T M}$ (chat, speech or video) was possible to give instructions to the caregiver or participant. Since sometimes the support could not help in overcoming some (mostly technical) errors with only verbal instructions, a remote takeover of the laptops was also possible. This was done via SSH and remote desktop under Linux. Finally, OpenVPN ${ }^{\mathrm{TM}}$ was used to remotely access laptops or to share certain resources (i.e. robot), even in environments with limited or restricted connectivity like clinics. 
Furthermore, we simplified the necessary steps and functionalities for the operators and designed a number of GUIs and scripts around our BCI to hide all the complexity. The following reduced functionality was finally provided:

- Viewing the raw EEG signals to check signal quality and to look for artifacts.

- Starting the BCI program, selecting the participant and choosing the mode (offline/online) or application.

- Transferring data between the local computer and BCI experts (server).

We want to point out, that during the reported experiments the infrastructure was only used to transfer data (EEG raw data and classifier configuration files) and to speak to the participants and therapists, but no remote takeover was necessary during any of the training sessions.

\subsection{User feedback and informal interviews}

During the whole process of transferring the BCI technology to clinics and end-users home and during the required adaptation process, we gathered a lot of data about problems with the current implementation and technology gaps. This information was not gathered via standard questionnaires, but on the basis of informal discussions, and on the experiences the caregivers, support persons, end-users and developers wanted to share with us. We asked very general and open questions to not influence or restrict the answers towards our phrased questions. During the analysis we grouped the experiences and problems along BCI related points and application related points. Similar statements were grouped together and phrased in a unified manner.

\section{Results}

In this section we first present the EEG features which have been identified for the various end-users and the achieved BCI control, before presenting the application performances. Furthermore, based on our experiences, we describe the lessons learned and problems encountered while transferring BCI technology towards end-user applications. 
Table 2: EEG channels (Chan.) and power spectral density (PSD) features (2 Hz band) used by each participant (ID) to control the BCI. Furthermore, the used motor imagery (MI) pair is given whereby "L" represents left hand, " $\mathrm{R}$ " right hand and "F" feet motor imagery. Participants indicated with ${ }^{*}$ had to be excluded during the training process because of inherent muscular artifacts due to their impairments.

\begin{tabular}{|c|c|c|c|c|c|c|c|}
\hline ID & Chan. & PSD band & MI & ID & Chan. & PSD band & MI \\
\hline \multirow[t]{2}{*}{$\mathrm{P} 1$} & $\mathrm{FCz}$ & $22 \mathrm{~Hz}$ & L-F & $\mathrm{P} 13$ & C3 & $12,14 \mathrm{~Hz}$ & $\mathrm{~L}-\mathrm{R}$ \\
\hline & $\mathrm{Cz}$ & $12,20,22 \mathrm{~Hz}$ & & & CP3 & $10,12 \mathrm{~Hz}$ & \\
\hline \multirow[t]{3}{*}{ P2 } & $\mathrm{C} 3$ & $10,12 \mathrm{~Hz}$ & L-R & & $\mathrm{CP} 1$ & $10,12 \mathrm{~Hz}$ & \\
\hline & $\mathrm{C} 2$ & $16 \mathrm{~Hz}$ & & $\mathrm{P} 14$ & $\mathrm{C} 1$ & $18,20 \mathrm{~Hz}$ & F-R \\
\hline & $\mathrm{C} 4$ & $10,12 \mathrm{~Hz}$ & & & CP1 & $8,10 \mathrm{~Hz}$ & \\
\hline \multirow[t]{2}{*}{ P3 } & $\mathrm{Cz}$ & $14,16,18,20,22 \mathrm{~Hz}$ & L-F & $\mathrm{P} 15$ & $\mathrm{FCz}$ & $24 \mathrm{~Hz}$ & F-R \\
\hline & $\mathrm{C} 4$ & $18,20,22,24 \mathrm{~Hz}$ & & & $\mathrm{FC} 2$ & $26,28 \mathrm{~Hz}$ & \\
\hline \multirow[t]{3}{*}{ P4 } & $\mathrm{FC} 4$ & $18,20 \mathrm{~Hz}$ & F-R & & $\mathrm{FC} 4$ & $14,16 \mathrm{~Hz}$ & \\
\hline & $\mathrm{C} 3$ & $8,10,20 \mathrm{~Hz}$ & & & $\mathrm{C} 2$ & $20 \mathrm{~Hz}$ & \\
\hline & $\mathrm{Cz}$ & $16,18,20 \mathrm{~Hz}$ & & & $\mathrm{C} 4$ & $20 \mathrm{~Hz}$ & \\
\hline \multirow[t]{4}{*}{ P5 } & C3 & $8,10 \mathrm{~Hz}$ & F-R & P16 & $\mathrm{FC} 1$ & $18 \mathrm{~Hz}$ & L-R \\
\hline & $\mathrm{C} 4$ & $8,10 \mathrm{~Hz}$ & & & C3 & $22,24,26 \mathrm{~Hz}$ & \\
\hline & CP3 & $8,10 \mathrm{~Hz}$ & & & $\mathrm{C} 4$ & $18,20 \mathrm{~Hz}$ & \\
\hline & CP4 & $8,10 \mathrm{~Hz}$ & & & $\mathrm{CP} 2$ & $20 \mathrm{~Hz}$ & \\
\hline \multirow[t]{2}{*}{ P6 } & $\mathrm{FCz}$ & $22,24,30 \mathrm{~Hz}$ & L-F & & $\mathrm{CP} 4$ & $18,20 \mathrm{~Hz}$ & \\
\hline & $\mathrm{FC} 4$ & $22,24,26 \mathrm{~Hz}$ & & $\mathrm{P} 17^{*}$ & $\mathrm{FCz}$ & $22,24,26 \mathrm{~Hz}$ & L-F \\
\hline P7 & $\mathrm{Cz}$ & $12,20,22,24 \mathrm{~Hz}$ & F-R & & C3 & $12 \mathrm{~Hz}$ & \\
\hline \multirow[t]{5}{*}{ P8 } & $\mathrm{Fz}$ & $22,24 \mathrm{~Hz}$ & L-R & & $\mathrm{Cz}$ & $26 \mathrm{~Hz}$ & \\
\hline & $\mathrm{FC} 1$ & $22,24,26 \mathrm{~Hz}$ & & & $\mathrm{CP} 3$ & $12,14,22 \mathrm{~Hz}$ & \\
\hline & $\mathrm{FC} 2$ & $22,24,26 \mathrm{~Hz}$ & & & $\mathrm{CP} 1$ & $20 \mathrm{~Hz}$ & \\
\hline & $\mathrm{C} 2$ & $24,26,28 \mathrm{~Hz}$ & & $\mathrm{P} 18$ & $\mathrm{FC} 4$ & $6,8,10,26,28 \mathrm{~Hz}$ & L-R \\
\hline & $\mathrm{C} 4$ & $24,26 \mathrm{~Hz}$ & & & $\mathrm{Cz}$ & $20,22 \mathrm{~Hz}$ & \\
\hline \multirow[t]{6}{*}{ P9 } & $\mathrm{FCz}$ & $10 \mathrm{~Hz}$ & $\mathrm{~L}-\mathrm{F}$ & & $\mathrm{C} 4$ & $6,8,10 \mathrm{~Hz}$ & \\
\hline & $\mathrm{FC} 2$ & $10,12 \mathrm{~Hz}$ & & P19 & C3 & $8,10,12 \mathrm{~Hz}$ & $\mathrm{~L}-\mathrm{F}$ \\
\hline & $\mathrm{FC} 4$ & $10 \mathrm{~Hz}$ & & P20 & FC3 & $10 \mathrm{~Hz}$ & $\mathrm{~L}-\mathrm{F}$ \\
\hline & $\mathrm{C} 2$ & $10,12 \mathrm{~Hz}$ & & & $\mathrm{FC} 2$ & $8,10,12,22 \mathrm{~Hz}$ & \\
\hline & $\mathrm{C} 4$ & $10,12 \mathrm{~Hz}$ & & & $\mathrm{FC} 4$ & $16,18,20,22 \mathrm{~Hz}$ & \\
\hline & $\mathrm{CP} 2$ & $10 \mathrm{~Hz}$ & & & $\mathrm{C} 4$ & $8,32,34 \mathrm{~Hz}$ & \\
\hline \multirow[t]{3}{*}{ P10 } & $\mathrm{FCz}$ & $18 \mathrm{~Hz}$ & L-F & & $\mathrm{CPz}$ & $10,12,14 \mathrm{~Hz}$ & \\
\hline & $\mathrm{C} 1$ & $18 \mathrm{~Hz}$ & & $\mathrm{P} 21^{*}$ & C3 & $12,14,16 \mathrm{~Hz}$ & F-R \\
\hline & $\mathrm{Cz}$ & $16,18,20 \mathrm{~Hz}$ & & & $\mathrm{Cz}$ & $16 \mathrm{~Hz}$ & \\
\hline \multirow[t]{4}{*}{ P11 } & $\mathrm{FC} 1$ & $20 \mathrm{~Hz}$ & L-R & & CP3 & $8,10 \mathrm{~Hz}$ & \\
\hline & $\mathrm{C} 2$ & $28 \mathrm{~Hz}$ & & P22 & C3 & $10,12 \mathrm{~Hz}$ & L-R \\
\hline & $\mathrm{CP} 2$ & $20,26 \mathrm{~Hz}$ & & & $\mathrm{C} 2$ & $12 \mathrm{~Hz}$ & \\
\hline & CP4 & $24,26 \mathrm{~Hz}$ & & & CP3 & $10,12 \mathrm{~Hz}$ & \\
\hline \multirow[t]{4}{*}{$\mathrm{P} 12$} & $\mathrm{Cz}$ & $10,30 \mathrm{~Hz}$ & L-F & $\mathrm{P} 23$ & FC3 & $18,20,22 \mathrm{~Hz}$ & F-R \\
\hline & $\mathrm{C} 4$ & $16,20 \mathrm{~Hz}$ & & & $\mathrm{Cz}$ & $20 \mathrm{~Hz}$ & \\
\hline & & & & & $\mathrm{CP} 3$ & $6,8 \mathrm{~Hz}$ & \\
\hline & & & & & $\mathrm{CPz}$ & $14 \mathrm{~Hz}$ & \\
\hline
\end{tabular}




\subsection{EEG features identified for the participants}

All participants started by imagining left hand, right hand and feet movements during calibration (offline) recordings. A classifier was then built for each pair of MI tasks. Table 2 presents the selected MI pair (highest controllability), and the corresponding EEG channels and power spectral density (PSD) features identified by the feature selection process, which were used online to control the BCI. We have ordered the participants in descending order of their final BCI online performance, independently of the time of recording, to increase the readability of the paper (therefore the participant with the best online performance would be P1. In the case of one participant (P24) we had technical problems with saving the raw EEG (which made the file unreadable for further analysis) and two participants (P17, P21) had to be excluded during the training process because of inherent muscular artifacts due to their impairments. Furthermore, participant P19 decided to stop participating in the study and dropped the recordings.

The MI pair mostly used was left hand versus feet (LF, 9 times), feet versus right hand (FR, 7 times) and left versus right hand (LR, 7 times). Therefore, in $70 \%$ of the cases feet imagery was involved. Looking at the subset of participants who tested the application this ratio is increasing to $80 \%(5 * \mathrm{LF}, 3 * \mathrm{FR}, 2 * \mathrm{LR})$. In general, the selected features are dominantly in the alpha band (around $10 \mathrm{~Hz}$ ) and in the beta band (around $22 \mathrm{~Hz}$ ), which is consistent with the literature $[21,22,18,1]$. Fig. 5 shows the histogram of the selected features and the corresponding electrode locations, for participants P1-P10 who tested the applications. Features were mostly chosen around $\mathrm{Cz}$ and $\mathrm{C} 4$, which is in line with the fact that most participants used left hand MI versus feet MI to control the BCI.

\subsection{BCI performance of online experiments}

Fig. 6 shows the performance of the online BCI runs using the Youden index for each participant, whereby $\mathrm{YI}=1$ means perfect control and 0 equals chance level. Participants printed in solid lines continued to the application testing, while participants in dashed lines did not produce any discriminable patterns or were excluded because of artifacts due to their impairments. Generally, ten participants showed very good BCI control $(Y I \geq 0.4)$ and tested the applications, additionally one participant (P18) showed a good performance of 0.64 during one single day, but was not able to reproduce it and the performance completely dropped afterwards without an identifiable 


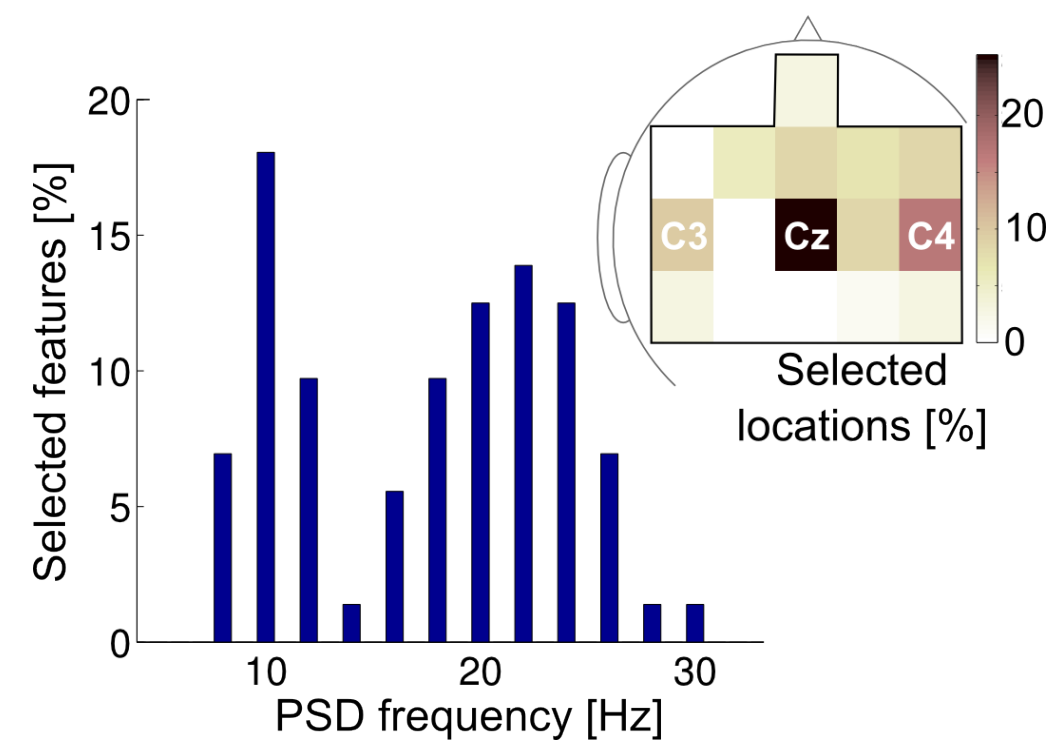

Figure 5: Histogram of the selected power spectral density (PSD) features for participants $\mathrm{P} 1-\mathrm{P} 10$ who tested the applications and the graphical representation of the corresponding channel locations.

reason. Furthermore, participant P11 did not achieve a high stable performance, although every second session reached up to $0.5-0.55$. Participant P9, which was one of the early participants, reported that he lost motivation since the pure BCI training was becoming boring for him and improved again when he was finally allowed to test the applications. Participants P4 and P6 had a holiday break in between the recordings. For end-users P5 and P8 we performed 3 online runs above the threshold of 0.4 , since their performance improvement was so incredible, that we wanted to check the stability first. Although the fluctuations over the different training sessions are quite large, a general improving trend is visible for participants (P1-P10), showing that these participants could improve their performance and modulate their brain patterns with practice.

Typically the mean trial times in the online runs per participants were between $2 \mathrm{~s}$ and $8 \mathrm{~s}$. Shortest trials went down to $0.76 \mathrm{~s}$ as the absolute minimum trial time restricted by the evidence accumulation. In some trials the participants needed a lot of time to deliver their commands and reached up to $40 \mathrm{~s}$. Such trial times are much too long and demanding. Therefore a trial timeout and a restart would be beneficial. 


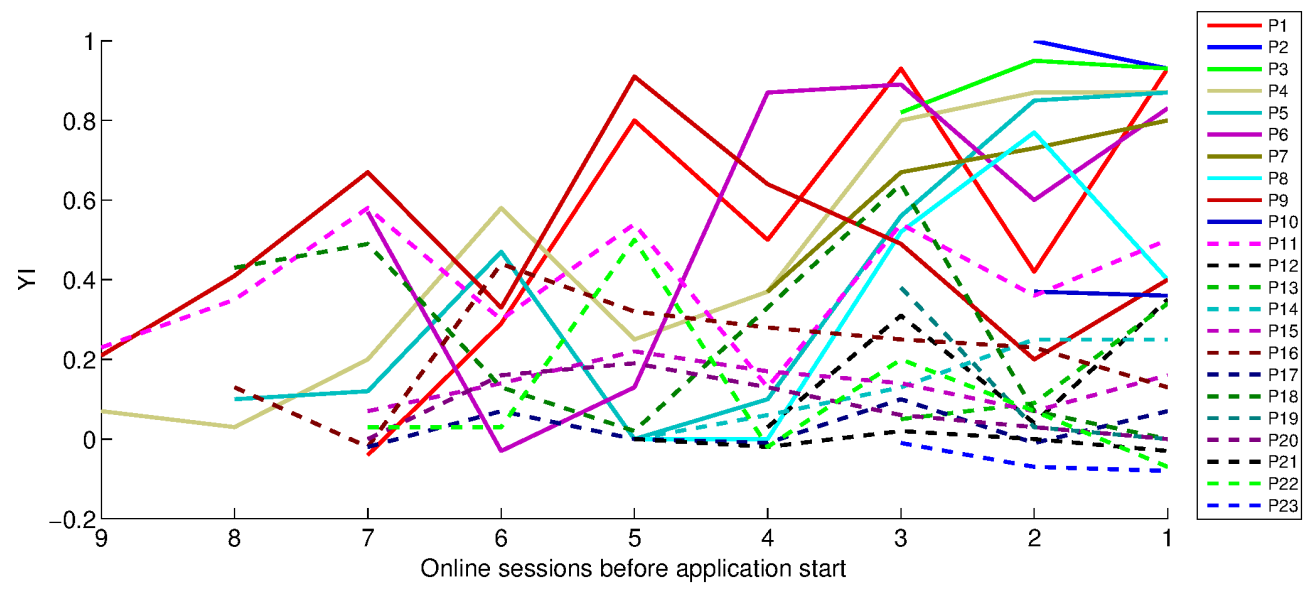

Figure 6: Performance values (Youden index, YI) of all online runs averaged per session for each participant (Participants printed in solid lines continued to the application testing).

\subsection{Application performance}

Ten end-users (P1-P10) fulfilled the requirements to test the applications. Since the whole experiment was limited to 10 days, not all participants could evaluate all applications. Nine participants had the time to operate the telepresence application and six the text entry application. All of them were able to successfully perform the tasks.

In Fig. 7 the performances values of the text entry application (character percentage) and of the tele-presence platform (ratio between the distances) are presented, both resulting in 1 for perfect and 0 for no control (same as the YI in the online runs).

In case of the tele-presence robot, participants achieved a mean ratio of $0.87 \pm 0.09$, with the best participant $\mathrm{P} 7$ achieving 0.97 , while the worst participant $\mathrm{P} 4$ still achieved 0.70 compared to the manual condition. The performance drop of participant $\mathrm{P} 4$ resulted from one single run, in which she intentionally delivered wrong commands believing that the target was somewhere else. The mean distance traveled to reach the targets was $12.7 \pm 1.5 \mathrm{~m}$ in a time of $96.0 \pm 12.4 \mathrm{~s}$. Remarkably, our end-users performed similar to the non-disabled users who were familiar with the environment, whose results were previously reported [23] with a mean time of $92.3 \pm 14.0 \mathrm{~s}$. Indeed, shared control helped all participants (including novel BCI participants or users with disabilities) to complete a rather complex task in similar time to 
those required by manual commands without shared control. More detailed results are given in $[23,15]$.

In case of the text entry application, the six participants achieved a mean of $0.93 \pm 0.05$, whereby participant P8 did not write any single wrong character. Typing speed varied across participants and words, due to fluctuating BCI performances, the fastest word trials were approaching 2 char $/ \mathrm{min}$, which is comparable to the performance achieved by P300 spellers [28, 29].

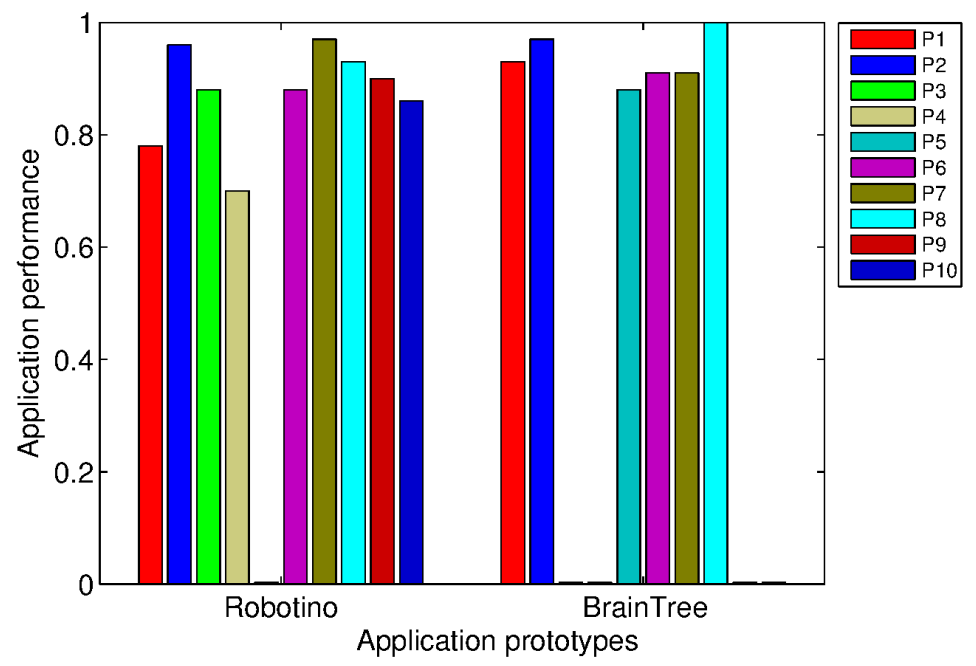

Figure 7: Application performances of the two applications (Robotino, Braintree) for the remaining subset of participants.

\subsection{Lessons learned and user feedback}

In this paper, we want to report especially our experiences, and the problems we encountered, while transferring our BCI from the lab to the clinics and to the end-user's home. This information was conducted from informal interviews with BCI experts, rehabilitation therapists in clinical institutions and the participating end-users. Tables 3 and 4 present the BCI-related and application-oriented issues, which were raised either by end-users (U), caregivers or therapists $(\mathrm{C})$ or by the BCI-experts (E). Some of the addressed points have already been improved in our current version (marked with a footnote in the Tables 3 and 4), since we were following a user-centered design and improved our system in several iterations. Nevertheless, we think 
it is important to mention them here, such that others can learn from them and avoid similar problems.

\section{Discussion}

The most important outcome is that all participants who achieved good BCI performances, could also control the applications successfully. Indeed, they were able to transfer the skill of "BCI control" from simple bar feedback to complex application prototype control. Although, we have to note that not all participants were able to learn to produce characteristic and stable EEG patterns, which could be used. Unfortunately, such results are in line with the literature [32]. In some subjects strong performance fluctuations over the training sessions occurred. There are various reasons for these fluctuations. The motivation of the participant is definitely a key factor, furthermore, slight differences in electrode cap placements can modify the produced brain patterns as well. So special care has to be taken considering these points.

Especially, whenever end-users reached a $Y I>0.6$, they mastered the applications equally well as healthy participants. This is very important, because having a good BCI control does not guarantee good control over the application, according to past experiences due to the necessary split attention between the application and the BCI (dual task). Another point which has to be considered is that BCI training does not require users to achieve $100 \%$ performance every trial, but most applications demand almost perfect performance all the time. If one or two trials during the training were performed erroneously, the overall performance is still okay, since each trial is more or less treated separately. In contrast, the impact of an error is critical in applications since one wrong decision needs a series of correct ones to overcome/correct the single error, which imposes heavy demands on users. Therefore, a better way to handle wrong decisions is required, either by means of an easy "undo" possibility [25] or smarter application designs. Unfortunately only $50 \%$ of the participants (10 out of 20 , if we remove the ones who stop or had too strong EMG artifacts) could test the applications. Due to the strict time limitations of our experimental protocol, we had to stop the training process of those end-users who did not reach a $Y \geq 0.4$ over two consecutive sessions after 10 days, although an increasing trend was visible in a few. The good application prototype control supports our claim, that shared control reduces participants' cognitive workload as it: (i) assists them in coping with low-level issues (such as obstacle avoidance in case of 
Table 3: Description of our experiences and problems encountered while transferring the BCI from the lab to the clinics/end-user's home, which were conducted from informal interviews ( $\mathrm{U}=$ end-user, $\mathrm{C}=$ caregiver or therapist, $\mathrm{E}=\mathrm{BCI}$-expert). Some of the mentioned points have already been tackled and implemented in our current version (marked with a footnote).

\begin{tabular}{|c|c|c|}
\hline \multicolumn{3}{|c|}{ BCI and training related points: } \\
\hline $\mathrm{a}$ & $\begin{array}{l}\text { Synchronized data folders for transferring the EEG data, classifier } \\
\text { and configuration files need good and stable internet connections. }\end{array}$ & $\mathrm{E}$ \\
\hline b & $\begin{array}{l}\text { The remote support infrastructure is helping in solving most tech- } \\
\text { nical problems. }\end{array}$ & $\mathrm{C}, \mathrm{E}$ \\
\hline c & $\begin{array}{l}\text { The BCI system consists of several components and cables which } \\
\text { have to be connected correctly, still too complex for non-experts. }{ }^{a}\end{array}$ & $\mathrm{C}$ \\
\hline$d$ & $\begin{array}{l}\text { It would be helpful if the caregiver/therapist has some technical } \\
\text { understanding about the BCI system (suggested already in [17]). }\end{array}$ & $\mathrm{E}$ \\
\hline e & $\begin{array}{l}\text { Adjusting simple parameters (e.g. thresholds) should be a quick } \\
\text { and easy process, so that on-site customisation can be done. }\end{array}$ & $\mathrm{U}, \mathrm{E}$ \\
\hline $\mathrm{f}$ & $\begin{array}{l}\text { BCI experts and therapists do not have the same background } \\
\text { knowledge and have a different (technical) vocabulary }[30,31] \text {. }\end{array}$ & $\mathrm{C}, \mathrm{E}$ \\
\hline $\mathrm{g}$ & $\begin{array}{l}\text { Many problems are triggered because of simple misunderstandings. } \\
\text { This issue is even stronger if the mother tongue is not used. }\end{array}$ & $\mathrm{U}, \mathrm{C}, \mathrm{E}$ \\
\hline $\mathrm{h}$ & $\begin{array}{l}\text { The instructions to the participants have to be given in his/her } \\
\text { mother tongue, to guarantee correct understanding. Furthermore, } \\
\text { different cognitive impairments should be taken into consideration. }\end{array}$ & $\mathrm{U}, \mathrm{C}$ \\
\hline $\mathrm{i}$ & $\begin{array}{l}\text { Mounting the electrodes by non-experts can take too much time } \\
\text { (up to } 1.5 \mathrm{~h} \text {, compared to } 15 \text { min by BCI experts) and contains too } \\
\text { many sources of error (floating electrodes, very high impedances, } \\
\text { misplaced caps). Active electrodes and pre-configured EEG caps } \\
\text { can reduce these issues and allow similar preparation times. }{ }^{b}\end{array}$ & $\mathrm{U}, \mathrm{C}$ \\
\hline $\mathrm{j}$ & $\begin{array}{l}\text { The training phase should be made more engaging and should pro- } \\
\text { vide more fun for the user, e.g. through game-like environments. }\end{array}$ & $\mathrm{U}$ \\
\hline $\mathrm{k}$ & $\begin{array}{l}\text { Highest motivation is achieved, if the end-user sees a personal } \\
\text { future need for the BCI. }\end{array}$ & $\mathrm{C}$ \\
\hline
\end{tabular}

${ }^{a}$ The first version of our BCI was not user-friendly enough. We have simplified our setup (e.g. reduced to 1 laptop, predefined caps instead of single electrodes, fewer connecting cables to overcome this issue.

${ }^{b}$ Using active electrodes with pre-configured caps reduced the preparation times down to the range of BCI experts; also misplaced caps and bad impedances vanished. 
Table 4: Continuation of Table 3: Description of our experiences and problems encountered while transferring the BCI from the lab to the clinics/end-user's home, which were conducted from informal interviews ( $\mathrm{U}=$ end-user, $\mathrm{C}=$ caregiver or therapist, $\mathrm{E}=\mathrm{BCI}$-expert). Some of the mentioned points have already been tackled and implemented in our current version (marked with a footnote).

Application and experiment related points:

1 Having a good BCI control does not guarantee good control over C,E the application, because an increased workload and split attention (dual task) between application and BCI feedback is required.

$\mathrm{m}$ Generally BCI training does not require users to achieve $100 \%$ U,E classification accuracy, but most applications demand almost perfect performance. The impact of an error is critical in applications since one wrong decision needs a series of correct ones to overcome/correct the error. A better way to handle wrong decisions is required, by means of either an easy "undo" possibility or smarter application designs. ${ }^{c}$

n Participants cannot deliver all BCI classes with the same easiness. Sometimes a bias towards one of the classes exists which yields in a strong performance deterioration. ${ }^{d}$

o BCI trainings are intended to improve the intentional control performance (delivering fast and accurate commands), but for most applications intentional-non control (INC) is more important which is not trained per se. ${ }^{e}$

p Extrapolating the last point, we can argue that most applications are using the BCI incorrectly, because they are forcing long "waiting" periods with many false positives, which yield frustration/stress that degrades the overall performance.

q Shared control and context awareness help the user to perform better and make it less demanding for them [23], especially in tasks with certain temporal precision.

$\mathrm{r}$ Participants mentioned that a "pause" mode would be beneficial, U otherwise BCI control can be too tiring for them.

${ }^{c}$ Such an effective error-handling mechanism is addressed by the hybrid BCI approach of the text-entry system [25]. Residual muscle activity allows the user to "undo" BCI actions. In case the user's level of disability does not allow this any longer, the normal backspace functionality can be used in a purely BCI-actuated fashion.

${ }^{d}$ Applying asymmetric or different thresholds for each class solves such a bias problem (see Section Appendix A.5).

${ }^{e}$ Under INC we understand the capability of not delivering unintended commands, e.g. the robot is moving forward. 
the robot or the language model in case of the text-entry, and is allowing the participant to focus the attention on his final destination) and thereby (ii) helps BCI users to maintain attention for longer periods of time (since the amount of BCI commands can be reduced and their precise timing is not so critical).

Besides the positive experiences and the promising results we have gained with the end-users, we have to acknowledge that a lot of work is still needed. Although we tried to hide the complexity of the BCI and of the prototype applications, our system is not ready to be used completely alone without our remote support at the end-user's place (as it is the case for most other BCI systems, especially motor imagery ones). This raises the question: How mature does BCI technology have to be before it can be given to end-users? Based on the user-centered design, our system has improved and has been simplified in several iterations and a lot of issues were be solved during the testing phase (c, e, i in Tables 3 and 4). Nevertheless, space for improvement exists in hardware, software, design and handling issues, before BCIs will become a commercial off-the-shelf product. The communication issues and language problems $(\mathrm{d}, \mathrm{g}, \mathrm{h})$ could be less important in other cases; e.g. if the end-user, the therapist and the BCI expert have all the same nationality, are all working in the same country and language region, which was not the case in our multi-national project.

We are aware that some of the points raised may seem trivial, but we were only able to identify them as truly recurrent problems as a result of the large number of end-user tests conducted outside the lab. In particular, points of Table 3 appeared because non-BCI experts took care of the recordings. Furthermore, some of the issues identified are also valid for other existing BCI implementations, so we anticipate that these issues may be faced similarly by other groups; especially if they try to bring their BCI technology to the enduser, to home environments and towards real application control. Therefore, we felt it important to raise awareness here.

"Floating" electrodes and bad impedances or even misplaced caps, should be automatically detected by intelligent algorithms in the future. Tracking changes of impedance (or signal quality) could be done during the recordings, either by special functions in EEG amplifiers or by analyzing the online changes in spectral components. Going even further, it will be soon possible to trace failed electrodes, and to replace them on the fly or to reconstruct their signals by looking at information from neighboring or related channels. Such an approach has already been demonstrated in an opportunistic network [33] 
and the first results with EEG will be available soon. Auto-configuration and fast auto-calibration through artificial intelligence, advanced signal processing and machine learning methods will further reduce the training time [34].

A very prominent source of disturbance, which creates a strong barrier for BCI users, is the fact that classifiers (independently of which one) are prone to develop a bias towards one BCI class. Either by shifted distributions or by sub-optimal decision hyperplanes, it becomes very difficult for the user to deliver one of the classes, which has a huge impact on the total performance. Online adaption [35] or online unbiasing [36] will soon facilitate the life of the user extremely, so that these changes can be followed during a recording and over sessions, making the delivery of both classes feasible all the time. Finally, smarter interface designs which are more robust to erroneous inputs, so that a single error should not cause the user a high workload to recover from it. This will help the user together with context awareness to improve the joint and final performance, although single controls will still be far from perfect. Such context aware systems, should adopt to the user's evolving capabilities and needs [37].

Furthermore, the difference between the outcome of a successful BCI training programme (intentional control) and the needs of the application (intentional non-control) became obvious. Such a possibility of entering in a non-control state becomes essential for mentally operating devices over long periods. Therefore, we actually suggest a change of the approach to controlling the applications and identified some possibilities: (i) Include INC in the training process [38]. (ii) Since a normal 2-class BCI is sometimes biased towards one class, we could exploit this natural bias. For instance, we could use the "hard" task for key commands (e.g., for selection). (iii) Design an "active-select" BCI: in the case of text entry or web browsing, the user makes the scan progress forward or backward by delivering mental commands. To select, the user stays in the INC state (which means that he does not deliver any commands) for a short period of time (akin to dwell-time in eye-tracking). (iv) Usage of multi-modal [39] or hybrid BCIs [2, 40, 41] where key commands (e.g., error corrections, pause, selection) are delivered through other channels such as residual muscular activity. As an example, the parallel monitoring of electromyographic activity from a single channel allows the user to "undo" one or more BCI actions through repetitive brisk movements. In case the user's level of disability or fatigue does not allow the use of this hybrid component, the existing backspace or undo functionality can be used instead in a purely BCI-actuated fashion [25]. 
To conclude, we want to mention explicitly that this paper aims to report the lessons learned, not possible mistakes in the operation of the BCIs, which are natural as with any other new advanced technology, requiring time to master it. Consequently, any limitation in the use of BCI technology remains mainly on our shoulders as researchers and developers, not on the users and caregivers. We want to use this opportunity to indulge the community in these important issues and share our, sometimes frustrating and other times amazingly encouraging experiences.

\section{Conclusion}

In this paper we investigated the issues of transferring BCI technology from BCI trainings with non-disabled participants towards end-users controlling applications. Data from 24 motor disabled end-users are presented, who were trained at their homes or clinics only by the therapists and caregivers, without the BCI experts present. The most important outcome is that fifty percent of the participants achieved good BCI performance and could successfully control the applications (tele-presence robot and text-entry system). Remarkably, our end-users performed similarly to the non-disabled users who were more familiar with the applications. They were able to (i) transfer the skill of "BCI control", which is very crucial, since having a good BCI control does not guarantee good control over the application, (ii) split their attention between the BCI task and the application and (iii) achieve application performances as good as healthy participants or even outperform them. We also shared our experiences and the lessons we learned during this technology transfer, which range from pure BCI problems (technical and handling), to common communication issues between different people involved, and lessons encountered while controlling the applications.

Altogether we could demonstrate that, modern human-computer interaction techniques combined with applications based on shared control and context awareness principles can be successfully controlled by a BCI and thereby providing powerful interactions and applications for disabled users. Furthermore, the performance of such applications can be improved by novel hybrid BCIs architectures, which are a synergistic combination of a BCI with other residual input channels. Our future work will focus on extending the clinical evaluation with more end-users, improved HCI aspects, advanced machine learning methods and adaptive BCI approaches in combination with hybrid BCIs. 


\section{Appendix A. Brain-computer interface details}

In this appendix the details about the underlying processes of the applied BCI to identify suitable brain features as a control signal are presented.

\section{Appendix A.1. Experimental paradigm}

During the training process (calibration recordings and online experiments), every trial starts with a fixation cross for 3 seconds on a screen in front of the participants (exact timing is given in Fig. A.8). Afterwards the cue - an arrow pointing to the left, right or up - is displayed for 5 seconds and the participants have to imagine repetitive kinaesthetic movements [42] with their left hand, right hand or feet depending on this cue. Since the participant is instructed by a cue, it is also called cue-based or synchronous BCI.

The output of the BCI is translated in a movement of the feedback bar (also called liquid cursor) and informs participants in online experiments about their current brain status. If the bar reaches the decision threshold, an additional discrete feedback in form of a large arrow (called decision) is presented to indicate which command is delivered and would be sent to the prototype in the case of application control. During the initial calibration recordings, where no online feedback is possible, the BCI output moves the feedback bar towards the correct side, so that the decision threshold is reached after 4 seconds. Every trial ends with a random pause of 3.0 to 4.5 seconds.

In total four offline runs (approximately $10 \mathrm{~min}$ each) with 15 trials for right, 15 trials for left and 15 trials for feet MI are recorded per participant, resulting in 60 trials per class for the classifier training. In the rare case that no classifier can be trained for this data, the offline runs are repeated in the next session.

In the online runs only two MI classes are used (the MI pair which is selected during the classifier training based on the highest controllability / performance). Each run consists of 15 trials each. In total 4-8 online runs are performed per session. Participants are pushed to move online as soon as possible, since the BCI feedback is a very important part of the training process $[43,13]$. So, whenever a classifier with an accuracy of more than $70 \%$ (equal to a $Y I=0.4$, see Section 2.4) could be identified, online BCI experiments are performed. At least 2 sessions of good online BCI control 
(with a $Y I \geq 0.4$, see Section 2.4) are requested before participants are allowed to test the applications (see time-line in Fig. 2).

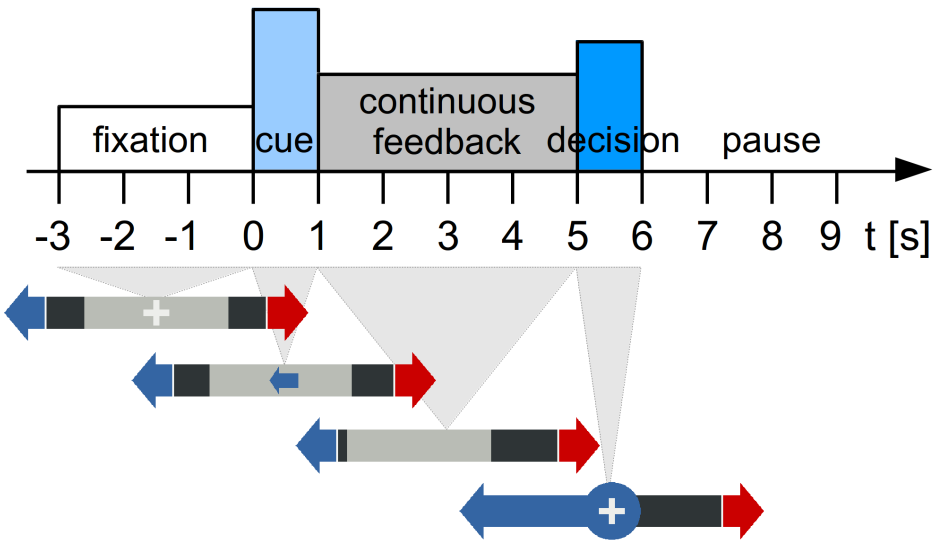

Figure A.8: Timing of a BCI trial (top) with corresponding screen visualizations (bottom). At second 0 a cue stimulus (in this case as a tiny blue arrow to the left) is given for 1 second and the light gray feedback bar starts moving accordingly to the BCI output in the online trials. If the bar reaches the threshold an additional discrete feedback in form of a large arrow -in this case as a blue arrow to the left- (called decision) is presented to indicate which command is delivered and sent to the application. The duration of the continuous feedback is fixed to $4 \mathrm{~s}$ in case of the offline runs, but has variable length in case of online runs, depending on the performance of the participant, typically between 2 and $8 \mathrm{~s}$.

\section{Appendix A.2. Feature extraction}

Each of the 16 EEG channels is spatially filtered with a Laplacian derivation whereby the weighted sum of the orthogonal neighboring channels is subtracted from each channel. Afterwards the power spectral density is estimated during the continuous feedback period for the frequency bands $4-48 \mathrm{~Hz}$ with $2 \mathrm{~Hz}$ resolution over the last second (resulting in 23 overall frequency components). The PSD is computed every $62.5 \mathrm{~ms}$ (i.e., 16 times per second) using the Welch method with 5 (75\% overlapping) internal Hanning windows of $500 \mathrm{~ms}$, resulting in 64 PSD calculations per trial. The feature extraction procedure yields an initial dimensionality $N=368$ of the feature vector (16 channels x 23 frequency components, where each individual feature reflects the estimated power of a specific cortical location (channel) and frequency. For the further processing steps the information at which time-point (inside 
the trial) the PSD is calculated is disregarded and the data of the different PSDs are pooled together.

\section{Appendix A.3. Feature selection}

To facilitate BCI control it is necessary to find those participant-specific spatial patterns that maximize the separability between the different mental tasks. From the initial 368 PSD features, we select, from the training dataset, a small subset (usually 5-10 features) so that the differences in mean PSD between the given number of classes are maximized, thus significantly reducing the dimensionality of the original feature vectors.

Our feature selection method is based on canonical variate analysis (CVA) in order to extract canonical discriminant spatial patterns (CDSP) which are the projections of the original PSD samples onto the canonical space [44]. Its output is a discriminant power (DP) metric for each PSD feature, which is used to rank all available features in terms of their contribution to the discriminability of the task-related brain patterns. Based on this ranking the final dimensionality $D$ of the feature vectors is determined either by keeping a predefined percentage of the overall DP or by explicitly selecting the $D$ highest ranking features. Fig. A.9 shows an exemplary map of the discriminant power and the features. The final selection is manually inspected to see which features have been selected, which provides very valuable information about the task the participants are doing, to see if new features are appearing over the training time and especially if the recorded data are contaminated by artifacts (in particular task-correlated artifacts, like inherent muscular activities). In our case the selected features of this purely data-driven method never contradicted prior neurophysiological knowledge concerning the cortical areas and frequency bands that are expected to be activated by the employed MI tasks.

\section{Appendix A.4. Classification}

Classification of the reduced PSD feature vectors is achieved using a Gaussian mixture model (GMM) framework, which outputs a conditional probability distribution $\vec{p}_{t}=\left[p_{t}^{1}, p_{t}^{2}, \ldots, p_{t}^{C}\right]$ at time $t$ over the $C$ mental tasks given each feature vector $\vec{x}_{t}$ [9]. Whereby, $t=0$ refers to the output timings of the feature extraction and classification which operates at $16 \mathrm{~Hz}$. Therefore, $t=0$ would be the arrival of the first sample in a trial and $t=1(62.5 \mathrm{~ms}$ later in real time) the arrival of the second sample, and so one. $t$ will increase within a trial until a decision is made (threshold reached). Each mental class 

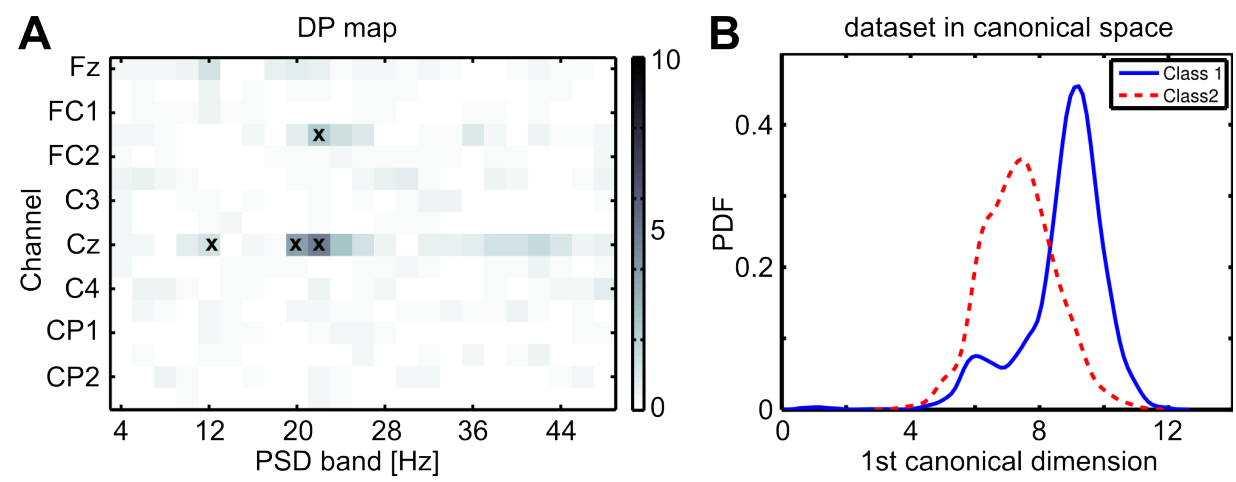

Figure A.9: (A) Map of discriminant power (DP) for each channel and frequency bin for participant P1. Selected features are marked with a "x". Note: Not all channels names are given for visualization purposes. (B) Projection of the selected features for the two selected motor imagery (MI) classes in CVA space.

is represented by a number of Gaussian units (usually $N=4$ ). The classconditional probability distribution function of class $i$ is a superposition of $N$ Gaussian prototypes. Equal priors for the classes and mixture coefficients are assumed, as well as shared, diagonal covariance matrices. The centroids of the Gaussian units are initialized by means of a self organizing map (SOM) clustering and their covariance matrices are subsequently computed as the pooled covariance matrices of the data closest to each prototype. Finally, the distribution parameters are, iteratively re-estimated through gradient descent so as to reduce the mean square error (MSE) [9]. The training of the Gaussian classifier stops, if the MSE change after each iteration is not improving, or after 20 iterations at maximum.

\section{Appendix A.5. Evidence accumulation}

Since the Gaussian classifier tends towards extreme (high and low) probabilities, using single-sample classifier evidence directly to drive the BCI feedback is likely to result in an fluctuating feedback and uncertain command delivery [9]. For these reasons, an evidence accumulation framework is embedded in our BCI, assisting in tackling uncertainty of the single-sample classifier output, providing smooth and informative feedback to the user, while at the same time ensuring flexibility towards the user needs due to the reconfigurability of the framework. Our implementation of evidence accumulation involves an exponential smoothing filter ("leaky" integrator), which 
preserves the fast refresh rate $(16 \mathrm{~Hz})$ of the classifier output $\vec{p}_{t}$. The output of the evidence accumulation module is a modified probability distribution over the mental classes $\vec{P}_{t}$, so that: $\vec{P}_{t}=\alpha \vec{P}_{t-1}+(1-\alpha) \vec{p}_{t}$, where $\alpha$ is a configurable, scalar, exponential smoothing factor. It controls the importance assigned to past evidences in comparison to the current one and, consequently, the trade-off between command delivery speed and accuracy. The modified probabilities $\vec{P}_{t}$ are visualized in real time, providing visual feedback to the user-i.e. movements of a feedback bar on the screen. A class $i$ type of command is delivered by thresholding the evidence accumulation output with class-dependent decision thresholds $t_{d_{i}}$, so that decision $=\max _{i}\left\{\vec{P}_{t}\right\}$, if $\max \left\{\vec{P}_{t}\right\}>t_{d_{i}}$.

It should also be noted, that in order to further filter out uncertain decisions, samples $x_{t}$ whose maximum element of the corresponding posterior probability distribution vector $\vec{p}_{t}$ does not exceed a rejection probability threshold $t_{r}$ are rejected and are not fed to the evidence accumulation framework at all (in which case the feedback bar stays still until $t+1$ ).

The contribution of this decision making scheme to the participants' online control of the BCI is two-fold. On the one hand, the smoothed final output, as illustrated through the continuous visual feedback bar, guides the participant into optimally modulating his brain activity to gradually reach the desired mental command, avoiding frustrating fluctuations. On the other hand, the application of the evidence accumulation framework is also critical for largely eliminating false positives during intentional non-control (INC) periods, while preserving the participants' ability to deliver intentional commands. By INC we mean the periods in which the participant is not wanting to deliver any command, e.g. waiting for the next selection step or waiting while a robot is moving forward (e.g. moving down a corridor).

Finally, the reconfigurability of parameters $\alpha, t_{d_{i}}, t_{r}$ allow for a BCI configuration specific to each individual user's needs and BCI training level. Typical values for the BCI hyper-parameters are $\alpha=0.96, t_{r}=0.6$ and $t_{d_{i}}=0.85$ for all classes $i$, but can be adjusted to each participants' preferences and needs.

\section{Appendix A.6. Feedback}

In the case of the online experiments, the output of the evidence accumulation directly moves the feedback bar and shows the participant its current status. The position of the bar is updated every $1 / 16$ of a second. If the bar reaches the decision threshold (see Fig. A.8), an additional discrete feedback 
in form of a large arrow (called decision) is presented to indicate which command is delivered and in the case of application control this would be sent to the prototype. The time to reach the threshold varies for every trial and depends only on the performance of the participant.

During the initial offline trials a faked BCI output moves the feedback bar towards the correct side, so that the decision threshold is reached after 4 seconds. The reason for the fake feedback is on the one hand to support the participant in the imagination task and on the other hand to simulate the same visual feedback behavior as for the runs with online feedback.

In both stages of this training process a synchronous BCI, also called cue-based BCI, is applied. Thereby, the participant is instructed by the cue which type of imagery he should perform. In case of the online experiments the feedback is based only on the participants brain patterns, and the time and type (MI class) of delivery depends only on them. The next logical step is to remove the cue (but leave the rest of the paradigm untouched) and let the participant decide what he wants to deliver. This can then be used to control an application or device at the users own pace. In such an asynchronous or un-cued BCI the performance cannot be evaluated directly, but indirectly by analyzing the overall goal of the brain controlled application.

\section{Acknowledgments}

This work is supported by the European ICT programme project TOBI: Tools for Brain-Computer Interaction (FP7-224631). This paper only reflects the authors' views and funding agencies are not liable for any use that may be made of the information contained herein. The authors would like to thank all patients and end-users for their participation, and all therapist and caregivers working on the different recording sites (Clinique Romande de Réadaptation-Suvacare, Switzerland; Klinik für Paraplegiologie, Universitätsklinikum Heidelberg, Germany; Associazione Italiana per l'Assistenza agli Spastici, Bologna, Italy) for their supporting work.

\section{References}

[1] Wolpaw J, Birbaumer N, McFarland D, Pfurtscheller G, Vaughan T. Brain-computer interfaces for communication and control. Clinical Neurophysiology 2002;113(6):767-791. 
[2] Millán JdR, Rupp R, Müller-Putz G, Murray-Smith R, Giugliemma C, Tangermann M, et al. Combining brain-computer interfaces and assistive technologies: State-of-the-art and challenges. Frontiers in Neuroscience 2010;4:161.

[3] Birbaumer N, Ghanayim N, Hinterberger T, Iversen I, Kotchoubey B, Kübler A, et al. A spelling device for the paralysed. Nature 1999;398(6725):297-298.

[4] Williamson J, Murray-Smith R, Blankertz B, Krauledat M, Müller KR. Designing for uncertain, asymmetric control: Interaction design for brain-computer interfaces. International Journal of Human Computer Studies 2009;67(10):827-841.

[5] Bensch M, Karim AA, Mellinger J, Hinterberger T, Tangermann M, Bogdan M, et al. Nessi: an EEG-controlled web browser for severely paralyzed patients. Computational Intelligence and Neuroscience 2007;71863:1-5.

[6] Riccio A, Leotta F, Bianchi L, Aloise F, Zickler C, Hoogerwerf EJ, et al. Workload measurement in a communication application operated through a P300-based brain-computer interface. Journal of Neural Engineering 2011;8(2):025028.

[7] Galán F, Nuttin M, Lew E, Ferrez PW, Vanacker G, Philips J, et al. A brain-actuated wheelchair: Asynchronous and non-invasive braincomputer interfaces for continuous control of robots. Clinical Neurophysiology 2008;119(9):2159-2169.

[8] Carlson T, Millán JdR. Brain-controlled wheelchairs: A robotic architecture. IEEE Robotics and Automation Magazine 2013;20(1):65-73.

[9] Millán JdR, Renkens F, Mouriño J, Gerstner W. Noninvasive brainactuated control of a mobile robot by human EEG. IEEE Transactions on Biomedical Engineering 2004;51(6):1026-1033.

[10] Bell CJ, Shenoy P, Chalodhorn R, Rao RPN. Control of a humanoid robot by a noninvasive brain-computer interface in humans. Journal of Neural Engineering 2008;5:214-220. 
[11] Lalor E, Kelly S, Finucane C, Burke R, Smith R, Reilly RB, et al. Steady-state VEP-based brain computer interface control in an immersive 3-d gaming environment. EURASIP Journal on Applied Signal Processing 2005;19:3156-3164.

[12] Nijholt A, Tan D, Allison B, Millán JdR, Graimann B, Jackson M. Brain-computer interfaces for HCI and games. In: Burnett M, Costabile M, Catarci T, de Ruyter B, Tan D, Czerwinski M, et al., editors. Proceedings ACM CHI. ACM Publishing, New York; 2008.

[13] Leeb R, Keinrath C, Friedman D, Guger C, Scherer R, Neuper C, et al. Walking by thinking: the brainwaves are crucial, not the muscles! Presence: Teleoperators and Virtual Environments 2006;15:500-514.

[14] Lotte F, Langhenhove AV, Lamarche F, Ernest T, Renard Y, Arnaldi B, et al. Exploring large virtual environments by thoughts using a braincomputer interface based on motor imagery and high-level commands. Presence: teleoperators and virtual environments 2010;19(1):54-70.

[15] Tonin L, Carlson T, Leeb R, Millán JdR. Brain-controlled telepresence robot by motor-disabled people. In: Proc. Annual International Conference of the IEEE Engineering in Medicine and Biology Society EMBC 2011. 2011, p. 4227-4230.

[16] Kübler A, Birbaumer N. Brain-computer interfaces and communication in paralysis: Extinction of goal directed thinking in completely paralysed patients? Clinical Neurophysiology 2008;119(11):2658-2666.

[17] Müller GR, Neuper C, Pfurtscheller G. Implementation of a telemonitoring system for the control of an EEG-based brain-computer interface. IEEE Transactions on Neural Systems and Rehabilitation Engineering 2003;11:54-59.

[18] Decety J. The neurophysiological basis of motor imagery. Behavioural Brain Research 1996;77(1-2):45-52.

[19] Ehrsson HH, Geyer S, Naito E. Imagery of voluntary movement of fingers, toes, and tongue activates corresponding body-part-specific motor representations. Journal of Neurophysiology 2003;90:3304-3316. 
[20] Jeannerod M, Frak V. Mental imaging of motor activity in humans. Current Opinion in Neurobiology 1999;9(6):735-739.

[21] Pfurtscheller G, Lopes da Silva FH. Event-related EEG/MEG synchronization and desynchronization: basic principles. Clinical Neurophysiology 1999;110:1842-1857.

[22] Pfurtscheller G, Neuper C. Motor imagery and direct brain-computer communication. Proceedings of the IEEE 2001;89:1123-1134.

[23] Tonin L, Leeb R, Tavella M, Perdikis S, Millán JdR. The role of sharedcontrol in BCI-based telepresence. In: Proc. of 2010 IEEE International Conference on Systems, Man and Cybernetics. 2010, p. 1462-1466.

[24] Schöner G, Dose M, Engels C. Dynamics of behavior: Theory and applications for autonomous robot architectures. Robotics and Autonomous Systems 1995;16:213-245.

[25] Perdikis S, Ramsey A, Leeb R, Williamson J, Al-Khodairy A, MurraySmith R, et al. Clinical evaluation of a hybrid-BCI text-entry system. In: Proceedings of TOBI Workshop III: Bringing BCIs to End-Users: Facing the Challenge. 2012, p. 75-76.

[26] Hu TC, Tucker AC. Optimal computer search trees and variablelength alphabetical codes. SIAM Journal on Applied Mathematics 1971;21(4):514-532.

[27] Youden WJ. Index for rating diagnostic tests. Cancer 1950;3(1):32-5.

[28] Guger C, Daban S, Sellers E, Holzner C, Krausz G, Carabalona R, et al. How many people are able to control a P300-based brain-computer interface (BCI)? Neuroscience Letters 2009;462(1):94-98.

[29] Sellers EW, Krusienski DJ, McFarland DJ, Vaughan TM, Wolpaw JR. A P300 event-related potential brain-computer interface (BCI): The effects of matrix size and inter stimulus interval on performance. Biological Psychology 2006;73(3):242-252.

[30] Riener R, Nef T, Colombo G. Robot-aided neurorehabilitation of the upper extremities. Medical and Biological Engineering and Computing $2005 ; 43: 2-10$. 
[31] Rosati G, Gallina P, Masiero S. Design, implementation and clinical tests of a wire-based robot for neurorehabilitation. IEEE Transactions on Neural Systems and Rehabilitation Engineering 2007;15(4):560 -569.

[32] Guger C, Edlinger G, Harkam W, Niedermayer I, Pfurtscheller G. How many people are able to operate an EEG-based brain-computer interface (BCI)? IEEE Transactions on Neural Systems and Rehabilitation Engineering 2003;11:145-147.

[33] Sagha H, Perdikis S, Millàn JdR, Chavarriaga R. Toward online detection of BCI performance degradation. In: Proceedings of TOBI Workshop III: Bringing BCIs to End-Users: Facing the Challenge. 2012, p. 89-90.

[34] Faller J, Vidaurre C, Solis-Escalante T, Neuper C, Scherer R. Autocalibration and recurrent adaptation: Towards a plug and play online ERD-BCI. IEEE Transactions on Neural Systems and Rehabilitation Engineering 2012;20(3):313-319.

[35] Vidaurre C, Kawanabe M, von Bünau P, Blankertz B, Müller K-R. Toward unsupervised adaptation of LDA for brain-computer interfaces. IEEE Transactions on Biomedical Engineering 2011;58(3):587-597.

[36] Perdikis S, Tavella M, Leeb R, Chavarriaga R, Millán JdR. A supervised recalibration protocol for unbiased BCI. In: Müller-Putz GR, Scherer R, Billinger M, Kreilinger A, Kaiser V, Neuper C, editors. 5th Int Brain-Computer Interface Conference, 2011. Graz, Austria: Verlag der Technischen Universität Graz; 2011, p. 8-11.

[37] Carlson T, Leeb R, Chavarriaga R, Millàn JdR. Online modulation of the level of assistance in shared control systems. In: Proceedings of the IEEE International Conference on Sytems Man and Cybernetics. 2012, p. 3321-3326.

[38] Zhang H, Guan C, Ang K.K, Chin ZY. Learning discriminative patterns for self-paced EEG-based motor imagery detection. Frontiers in Neuroscience 2012;6(7).

[39] Leeb R, Lancelle M, Kaiser V, Fellner D, Pfurtscheller G. Thinking Penguin: Multi-modal Brain-Computer Interface Control of a VR Game. 
IEEE Transactions on Computational Intelligence and AI in Games 2013;5(2):117-128.

[40] Leeb R, Sagha H, Chavarriaga R, Millán JdR. A hybrid brain-computer interface based on the fusion of electroencephalographic and electromyographic activities. Journal of Neural Engineering 2011;8(2):025011.

[41] Müller-Putz G, Breitwieser C, Cincotti F, Leeb R, Schreuder M, Leotta F, et al. Tools for Brain-Computer Interaction: a general concept for a hybrid BCI. Frontiers in Neuroinformatics 2011;5:30.

[42] Neuper C, Scherer R, Reiner M, Pfurtscheller G. Imagery of motor actions: differential effects of kinaesthetic versus visual-motor mode of imagery on single-trial EEG. Brain Research Cognitive Brain Research 2005;25:668-677.

[43] Kübler A, Neumann N. Brain-computer interfaces - the key for the conscious brain locked into a paralyzed body. Progress in Brain Research 2005;150:513-525.

[44] Galán F, Ferrez PW, Oliva F, Guàrdia J, Millàn JdR. Feature extraction for multi-class BCI using canonical variates analysis. In: IEEE International Symposium on Intelligent Signal Processing. 2007. 